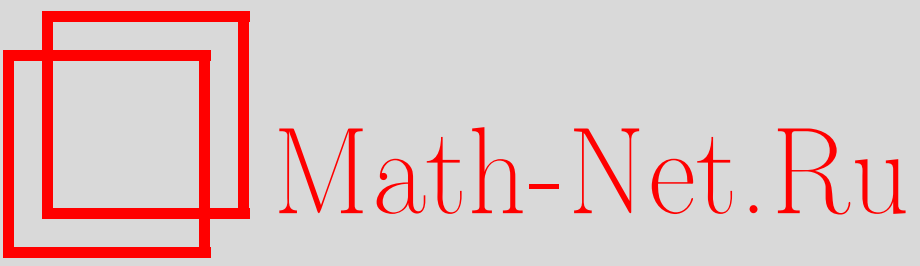

В. Э. Турчин, Гомологии комплексов двусвязных графов, УМH, 1997, том 52, выпуск 2, 189-190

DOI: https://doi.org/10.4213/rm845

Использование Общероссийского математического портала Math-Net.Ru подразумевает, что вы прочитали и согласны с пользовательским соглашением

http://www.mathnet.ru/rus/agreement

Параметры загрузки:

IP : 3.85 .5 .30

26 апреля 2023 г., 13:54:41 


\section{ГОМОЛОГИИ КОМПЛЕКСОВ ДВУСВЯЗНЫХ ГРАФОВ}

\section{В.Э. Турчин}

В работе доказано, что гомологии комплексов двусвязных графов нетривиальны только в размерности $2 n-4$, и их ранг равен $(n-2)$ !. Гомологии этих комплексов представляют существенный интерес в вычислении гомологий пространств узлов в $\mathbb{R}^{n}, n \geqslant 3$, см. [5]. Этот же резултат почти одновременно был получен с использованием принципиально иной техники группой математиков [2].

Пусть $M_{n}$ - множество точек, занумерованное числами $1,2, \ldots, n$. Мы будем рассматривать графы с вершинами из множества $M_{n}$ без петель и кратных ребер. Связньй граф̆ назьвается двусвязным, если при удалении из него любой вершины он не теряет связность.

Сопоставим множеству $M_{n}$ симплекс с $n(n-1) / 2$ вершинами. Каждой вершине симплекса соответствует отрезок, соединяющий пару различных точек нашего множества. Тогда каждому граф̆у (с непустьм множеством ребер) на $M_{n}$ отвечает грань симплекса, вершины которой суть ребра граф̆а.

Объединение всех граней, соответствующих несвязньм (не двусвязным) графрам, является симплициальным комплексом. Рассматривая факторы комплекса всех граней симплекса по этим подкомплексам, получаем комплексы $\Delta_{n}, \Delta_{n}^{2}$ связных (соответственно, двусвязных) графовов.

Пусть $G$ - произвольная (абелева) группа коэффициентов.

Лемма 0 [4], [1], [3]. $H_{i}\left(\Delta_{n}, * ; G\right)=0$ npu $i \neq n-2, u H_{n-2}\left(\Delta_{n}, * ; G\right)=G^{(n-1) !}$.

TEOpema. $H_{i}\left(\Delta_{n}^{2}, * ; G\right)=0$ npu $i \neq 2 n-4 u H_{2 n-4}\left(\Delta_{n}^{2}, * ; G\right)=G^{(n-2) !}$.

ДокАЗАТЕЛЬСтво теОРемы. Рассмотрим фильтрацию в комплексе $\Delta_{n}^{2}: X_{1} \subset X_{2} \subset \cdots \subset$ $X_{n-1}=\Delta_{n}^{2}$, где $X_{i}$ порождено графами с не более чем $i$ ребрами, выходящими из точки $\{1\} \in M_{n}$

ЛЕМма 1. $H_{j}\left(X_{i}, X_{i-1} ; G\right)=0$, еслu $i \neq n-1, j \neq 2 n-4, u H_{2 n-4}\left(X_{n-1}, X_{n-2} ; G\right) \simeq$ $G^{(n-2) !}$

Теорема есть очевидное следствие этой леммы.

ДОКАЗАТЕЛЬСТВО ЛЕМмЫ 1 . Если $i=n-1$, то $X_{n-1} / X_{n-2}$ будет комплексом, состоящим из двусвязных грабоов с $(n-1)$ ребром, выходящим из первой вершины.

ПредЛОЖЕНИЕ 1. Граф, в котором первая вершина соединена со всеми оставшимися, двусвязен тогда и только тогда, когда его подграф, состоящий из всех точек кроме точки 1, связен.

Доказательство предложения 1 очевидно.

СлЕДСтвиЕ. $H_{*}\left(X_{n-1}, X_{n-2} ; G\right) \simeq H_{*-(n-1)}\left(\Delta_{n-1}, * ; G\right)$.

Применяя лемму 0 , получаем утверждение теоремы при $i=n-1$.

Обозначим через $R_{i}(n)$ подкомплекс комплекса $X_{i} / X_{i-1}$, соответствующий граффам, в которых точка 1 соединяется только с точками $2,3, \ldots, i+1$.

Тогда $H_{*}\left(X_{i}, X_{i-1} ; G\right) \simeq\left(H_{*}\left(R_{i}(n), * ; G\right)\right)^{\left(\begin{array}{c}n-1 \\ i\end{array}\right)}$, так как $X_{i} / X_{i-1}$ является букетом $\left(\begin{array}{c}n-1 \\ i\end{array}\right)$ изоморффных $R_{i}(n)$ комплексов.

ПРЕДЛОЖЕНИЕ $2 . H_{*}\left(R_{i}(n), * ; G\right)=0($ nрu $0<i<n-1)$.

Работа выполнена при финансовой поддержке Российского фонда фундаментальных исследований (грант № 95-01-00846а). 
ДоКАЗАТЕЛЬСТво ПРЕДЛОЖЕНИЯ 2. Будем доказьвать индукцией по $i$.

Пусть $i=1$, тогда это очевидно, так как подобных графов просто не существует.

Предположим, что это доказано для всех $i=1,2, \ldots, k-1$, и докажем это для $i=k>1$.

ОПРЕДЕЛЕниЕ 1. Ребро двусвязного графа принципиально, если для этого ребра граф̆ перестает быть двусвязньгм.

ОПРЕДЕлЕниЕ $1^{\prime}$. Принципиальное ребро двусвязного граффа принципиально относительно точки $p$, если без этого ребра и без точки $p$ граф̆ перестает быть связным.

Рассмотрим следующую фильтрацию на комплексе $R_{k}(n)$ : граф̆ принадлежит члену $Y_{i}$ фильтрации, если в нем содержится $\leqslant i$ ребер, не совпадающих с ребром $(2,3)$. Диффференциал $d_{0}$ спектральной последовательности, определенной по этой филштрации, действует нулем на графах, не содержащих ребро $(2,3)$, либо же, если оно есть, удаляет его. В частности, первый член $E^{1}$ этой спектральной последовательности состоит из графов, в которых ребро $(2,3)$ принципиально. Итак, используя эту спектральную поверхность, мы получаем, что гомологии комплекса $R_{k}(n)$ изоморфны гомологиям своего подкомплекса, состоящего только из графов, в которых ребро $(2,3)$ принципиально. Этот подкомплекс есть букет $2^{n-3}$ комплексов. Действительно, ребро $(2,3)$ любого такого граффа $\Gamma$ может быть принципиалњным толњко относительно точки 1 (так как $\Gamma$ содержит ребра $(1,2)$ и $(1,3)$ ), что означает разбиение множества точек $4,5, \ldots, n$ на два подмножества: $M_{2}$ и $M_{3}$, где $M_{2}$ (соответственно, $\left.M_{3}\right)$ точки, которые последовательностью ребер, не совпадающих с ребром $(2,3)$ и не проходящих через первую вершину, можно соединить с точкой 2 (соответственно, с точкой 3 ).

Для всякого разбиения множества $\{4,5, \ldots, n\}$ на два подмножества $M_{2}$ и $M_{3}$ мы получаем некоторый комплекс. Мы докажем, что каждый такой комплекс ацикличен.

Рассмотрим произвольньй граф̆, имеющий ребра $(1,2),(1,3),(2,3)$ и такой, что его подграф, получающийся удалением из него вершины $\{1\}$ и ребра $(2,3)$, имеет две связные компоненты $M_{2} \cup\{2\}$ и $M_{3} \cup\{3\}$.

УТВеРЖДЕНИЕ. Такой граф двусвязен тогда и только тогда, когда оба подграфа, порожденные вершинами $\{1\} \cup\{2\} \cup M_{2}$ (соответственно, $\{1\} \cup\{3\} \cup M_{3}$ ), двусвязны.

Утверждение доказьвается непосредственной проверкой.

СледСтВИЕ. Любой подкомплекс (из $2^{n-3}$ возможных) гомотопически әквивалентен двукратной надстройке произведения $R_{k_{2}}\left(n_{2}\right) \times R_{k_{3}}\left(n_{3}\right)$, әде $k_{2}+k_{3}=k, n_{2}+n_{3}=n+1$ $\left(k_{i} \geqslant 1, k_{i}<n_{i}\right)$. Но $k<n-1$, следовательно, существует $i \in\{2,3\}: k<n_{i}-1$, а значит, $H_{*}\left(R_{k}\left(n_{i}\right), * ; G\right)=0$ (по предположению индукции). Таким образом, $H_{*}\left(\Sigma^{2}\left(R_{k_{2}}\left(n_{2}\right) \times\right.\right.$ $\left.\left.R_{k_{3}}\left(n_{3}\right)\right), * ; G\right)=0$.

ЗАмЕчАНиЕ. Метод доказательства предложения 2 применим и для вычисления гомологий комплекса связных графов (лемма 0).

\section{СПИСОК ЛИТЕРАТУРЫ}

[1] Björner A. // Handbook of Combinatorics / ed. R. Graham, M. Grötshel and L. Lovász. Amsterdam: North-Holland, 1995. P. 1819-1872. [2] Babson E., Björner A., Linusson S., Shareshian J., Welker V. Complexes of not $i$-connected graphs - extended abstract (to appear). [3] Björner A., Walker J. W. // European J. Combin. 1983. V. 4. № 1. P. 11-19. [4] Vassiliev V. A. // The I. M. Gelfand's Seminars / ed. L. Corwin. Boston: Birkhäuser, 1993. P. 223-235. [5] Васильев В. А. Топология дополнений к дискриминантам. М.: Фазис, 1997. 